\title{
Zika virus crosses an in vitro human blood brain barrier model
}

\author{
Judie B. Alimonti ${ }^{1}$, Maria Ribecco-Lutkiewicz ${ }^{2}$, Caroline Sodja², Anna Jezierski ${ }^{*} \mathbb{E}$, Danica B. Stanimirovic ${ }^{2}$, \\ Qing Liu², Arsalan S. Haqqani' ${ }^{1}$ Wayne Conlan ${ }^{1}$ and Mahmud Bani-Yaghoub ${ }^{2}$
}

\begin{abstract}
Zika virus (ZIKV) is a flavivirus that is highly neurotropic causing congenital abnormalities and neurological damage to the central nervous systems (CNS). In this study, we used a human induced pluripotent stem cell (iPSC)-derived blood brain barrier (BBB) model to demonstrate that ZIKV can infect brain endothelial cells (i-BECs) without compromising the BBB barrier integrity or permeability. Although no disruption to the BBB was observed post-infection, ZIKV particles were released on the abluminal side of the BBB model and infected underlying iPSC-derived neural progenitor cells (i-NPS). AXL, a putative ZIKV cellular entry receptor, was also highly expressed in ZIKV-susceptible i-BEC and i-NPs. This iPSC-derived BBB model can help elucidate the mechanism by which ZIKV can infect BECs, cross the BBB and gain access to the CNS.
\end{abstract}

Keywords: Zika virus, Brain endothelial cells, Blood-brain barrier model, iPSC, AXL, Neural progenitors

\section{Introduction}

Zika virus (ZIKV), a mosquito-borne flavivirus, was generally perceived to cause mild infections in adults. However, the 2015-2016 ZIKV outbreak in Brazil has been associated with increased cases of microcephaly, Guillain-Barré syndrome and other neurological conditions $[1,2]$. There is substantial evidence that ZIKV is neurotropic (reviewed in [3]) since it is routinely found in brains of microcephalic infants [4] and human brain organoids [5]. ZIKV also shows tropism for the central nervous system (CNS) of mice [6,7]. Neonatal ZIKV infection leads to abnormal vascular density and diameter in the developing brain, resulting in a leaky bloodbrain barrier (BBB) and massive neuronal death [8]. Recent evidence suggests that ZIKV is also neurotropic in adults $[9,10]$ raising the possibility that the intact $\mathrm{BBB}$ can be 'permissive' to ZIKV infection allowing access to brain cells. In this study, a novel in vitro stem-cell derived BBB model was used to evaluate the ability of a 2013

\footnotetext{
*Correspondence: anna.jezierski@nrc-cnrc.gc.ca

${ }^{2}$ Human Health Therapeutics Research Center, National Research Council of Canada, Bldg M54-1200 Montreal Rd., Ottawa, ON K1A 0R6, Canada Full list of author information is available at the end of the article
}

ZIKV isolate (Asian lineage) [11] to cross the BBB and infect human neural cells.

The BBB is a tightly sealed and specialized endothelial cell layer which prevents many drugs, particulates and microbes from entering the brain. BBB models in vitro have been used routinely to evaluate the mechanisms of passage of therapeutics and viruses across the BBB [1215]. To overcome limitations inherent in human primary and immortalized brain endothelial cell (BEC) models, including low baseline transendothelial electrical resistance (TEER), discontinuous tight junctions and fast loss of the BBB phenotype in culture [16], BBB models differentiated from human stem cells have been developed [17]. Recently, we have described [18] a human BBB model based on a two-step differentiation protocol of induced pluripotent stem cells (iPSCs) derived from amniotic fluid cells into induced brain endothelial cells (i-BECs), as well as into neural progenitor cells (i-NPs) and mature neurons (i-Ns) (Additional file 1: Figure S1). This i-BEC model has been shown to recapitulate the salient molecular and functional features of the $\mathrm{BBB}$, including the expression of BBB-enriched transporters and receptors, high transendothelial electrical resistance (TEER) and the ability to discriminate species-selective mechanisms of receptor-mediated antibody transport 
[18]. Here, we provide a demonstration of this model's utility in evaluating viral invasion across the BBB coupled with viral infection of target brain cells.

\section{Results}

Initially, to confirm that ZIKV could infect human i-NPs and i-Ns, the virus was added directly to these cells for up to 72 h (Fig. 1). i-NPs were highly susceptible (Fig. 1a, b), whereas terminally differentiated iNs were much less permissive to ZIKV infection (Fig. 1c, d; quantified in Fig. 1e). The receptor tyrosine kinase AXL, a putative entry receptor that mediates endocytosis of flaviviruses into host cells $[19,20]$ has been proposed as a

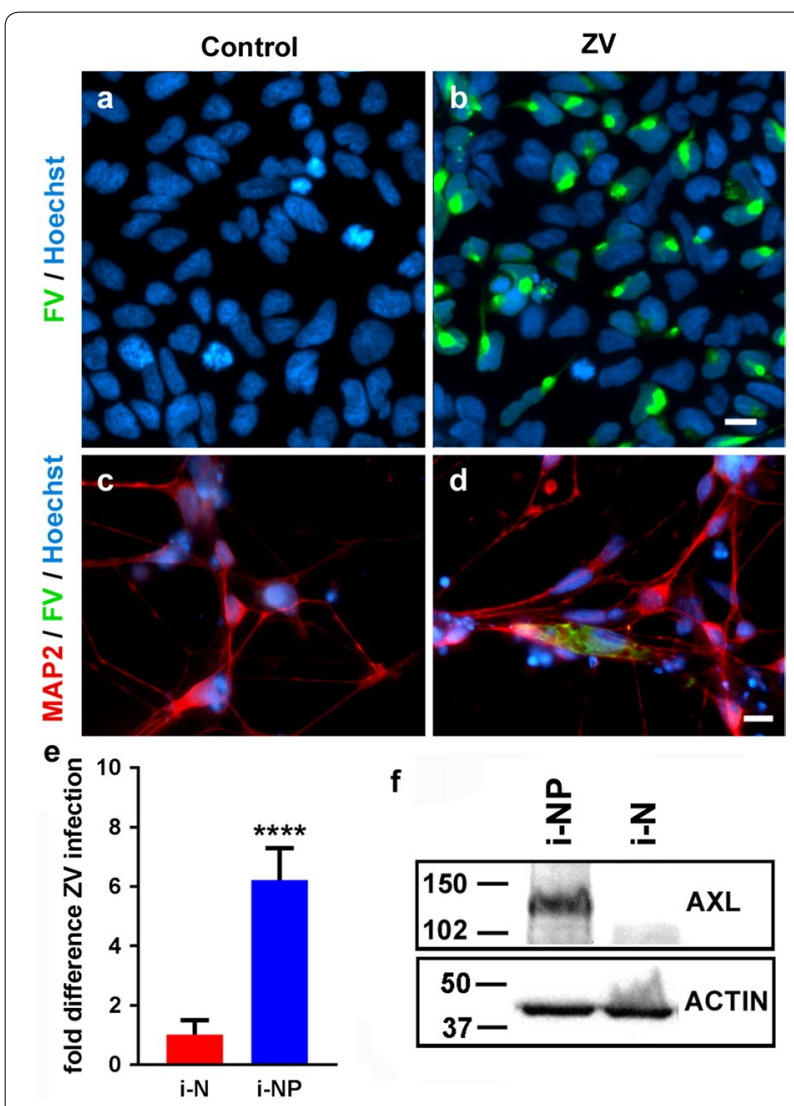

Fig. 1 Direct ZIKV infection and AXL expression in i-NP and i-Ns. a-d ZIKV infection was detected in the cytoplasm of $\mathbf{b}$ i-NPs and $\mathbf{d}$ i-Ns by immunofluorescence staining $72 \mathrm{~h}$ after $\mathrm{ZIKV}$ infection $(\mathrm{MOI}=4)$. The nuclei were stained with Hoechst dye (blue) and the virus was detected using anti-flavivirus group antibody (FV; green). i-Ns were additionally stained with the anti-MAP2 (red) antibody. Scale bar $=10 \mu \mathrm{m}$. e Fold difference of ZIKV (ZV) infection in i-NP, relative to $\mathrm{i}-\mathrm{Ns}$, based on cell counts of FV positive cells (mean \pm SD). Cell counts are from two independent experiments, six fields counted, with at least 80 cells/field. $\left({ }^{* * *} p<0.0001\right.$, Student's T test). $\mathbf{A}$ representative Western blot of putative ZIKV receptor AXL expression in i-NPs and i-Ns detected by an anti-AXL antibody. $\beta$-ACTIN was used as an internal loading control candidate ZIKV entry receptor in human glial cells [21], neural stem cells [19] and radial glial cells [19]. The putative ZIKV receptor AXL was also highly expressed in the i-NPs but not in i-Ns (Fig. 1f), which correlated with the lower infection of i-Ns observed in this study (Fig. 1e) and as previously described [22, 23].

Similarly, ZIKV infection of the i-BECs was identified $24 \mathrm{~h}$ post-exposure to the virus by immunofluorescence (Fig. 2a, b) and was detected in cell lysates by Western blotting after only a 2-h exposure (Fig. 2c). ZIKV-infected i-BEC monolayers remained viable with no visible compromise to the integrity and continuity of their intercellular contacts, as assessed by their staining for a tight junction protein, ZO-1 (Fig. 2b). Putative ZIKV receptor AXL was highly expressed in i-BECs by Western blot analysis (Fig. 2d) as well as in human brain endothelial cell line hCMEC/D3 by LC-MS (Fig. 2e). In hCMEC/D3 cells, AXL expression levels were similar to those of SLC2A1 (GLUT1), and higher than the expression of two other putative ZIKV receptors MERTK and TYRO3 (which was not detectable) (Fig. 2e). $A X L$ was also identified as a highly abundant transcript in primary human and rat brain endothelial cells by NGS analyses (Fig. 2f) and was highly enriched in isolated rat and mouse brain microvessels, compared to whole brain tissues (Fig. 2f). In contrast to low expression of MERTK and TYRO3 transcripts in brain endothelial cells (Fig. 2e, f), both MERKT and TYRO3 were expressed in isolated brain microvessels and total brain extracts (Fig. 2f). AXLmediated infection by ZIKV has been shown in a variety of endothelial cells [24] including human umbilical vein endothelial cells (HUVECs) [25] and could play a role in ZIKV infection of brain endothelial cells, and specifically, i-BECs.

To determine whether ZIKV could cross the BBB, virus was added into the apical compartment of the Transwell inserts containing i-BEC monolayers. The inserts were then placed in a 12 well companion plate containing adherent i-NPs (Additional file 1: Figure S1). If ZIKV crossed the BBB, the virus would be present in the fluid compartment between the two cellular layers resulting in i-NP infection.

To assess BBB integrity in i-BECs during ZIKV exposure, two parameters were examined: TEER, prior to infection, and sodium fluorescein permeability postinfection. In this BBB model, TEER values have been shown to closely inversely correlate with sodium fluorescein paracellular permeability [18]. Following infection of the i-BECs, the BBB paracellular permeability remained unchanged, ascertained by sustained restriction of sodium fluorescein passage in infected $\left(0.10 \pm 0.05 \times 10^{-3} \mathrm{~cm} / \mathrm{min}\right)$ compared to mock-infected controls $\left(0.09 \pm 0.05 \times 10^{-3} \mathrm{~cm} / \mathrm{min}\right)$ (Fig. 3a). At the 

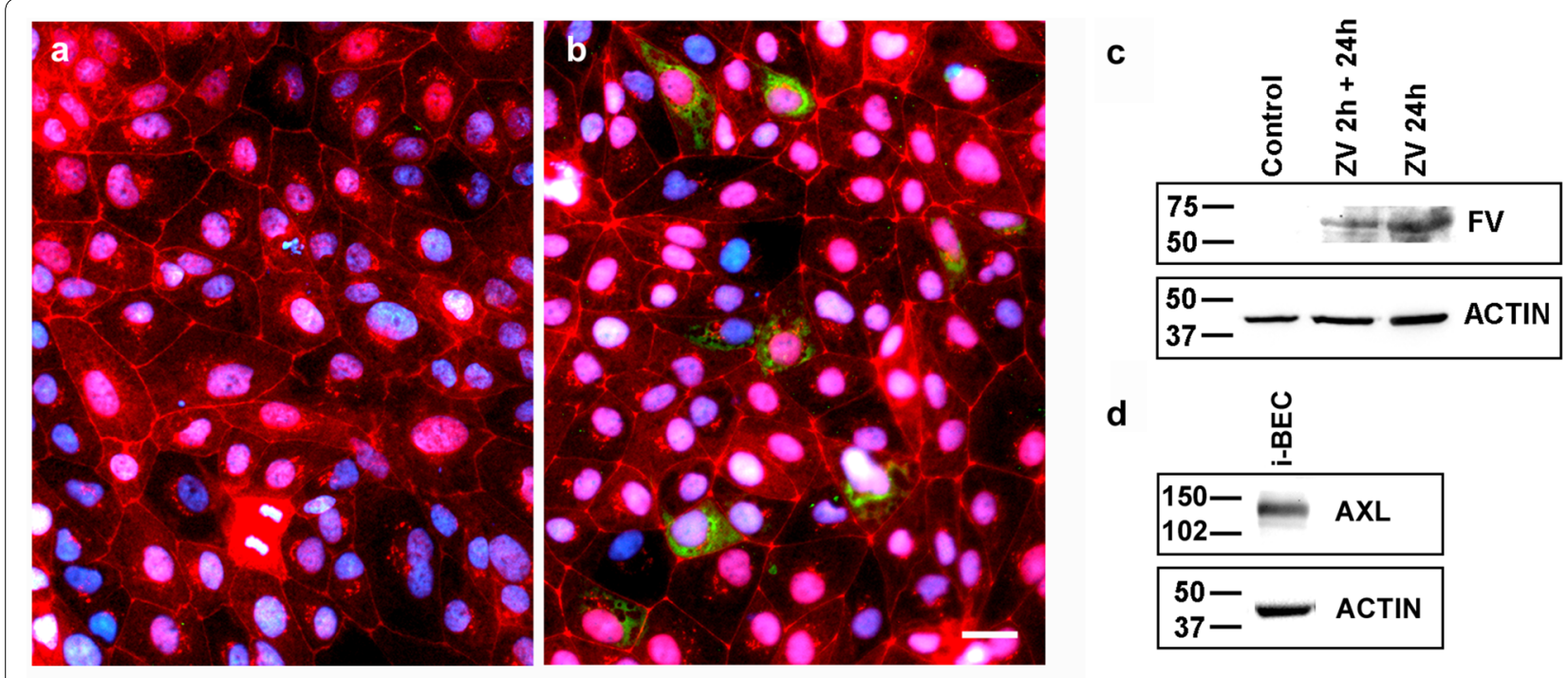

d

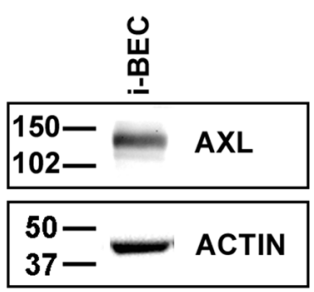

e

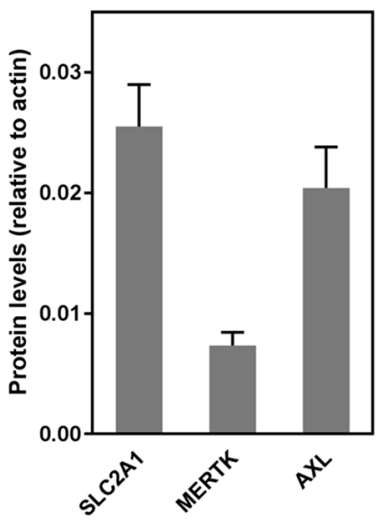

f

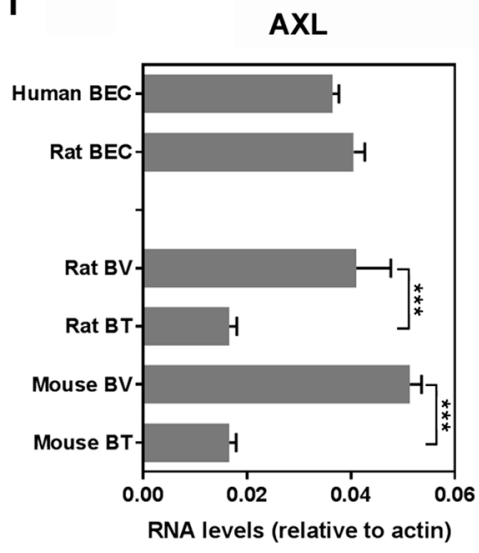

MERTK

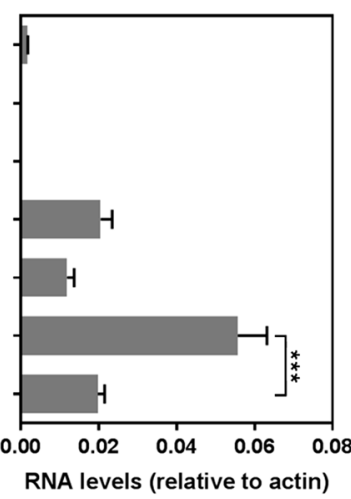

TYRO3

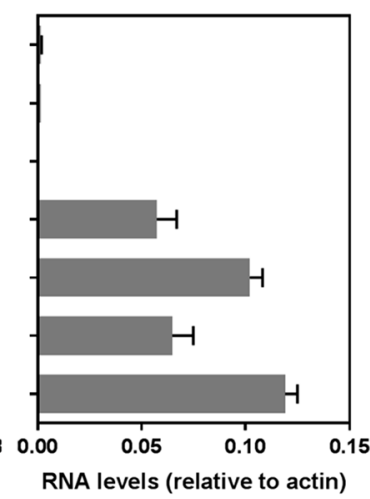

Fig. 2 ZIKV infection and AXL expression in BECs. a, b Immunofluorescence staining was conducted 24 h post ZIKV infection (MOI=4) of i-BECs using anti-flavivirus group antibody (FV; green). ZIKV was detected in infected i-BECs (b) but not in uninfected controls (a). Tight junction marker ZO-1 was used to label cell-cell contacts (red); nuclei were stained using Hoechst dye (blue). Scale bar = $10 \mu \mathrm{m}$. c The presence of ZIKV in i-BECs was also determined by Western blotting at 2 or $24 \mathrm{~h}$ after exposure to the virus. $\mathbf{d}$ Representative Western blot demonstrating putative ZIKV receptor $A X L$ expression in i-BECs using an anti-AXL antibody. $\beta$-ACTIN was used as an internal loading control. e Expression levels of SLC2A1 (GLUT-1), MERTK and AXL in human brain endothelial cell line hCMEC/D3 determined by proteomics (LC-MS). Shown are ratio of intensity-based absolute quantification values (mean \pm SD from 3 independent cell preparations) calculated for each protein and $\beta$-ACTIN, as described previously [41]. f The expression of AXL, MERTK and TYRO3 in primary human and rat brain endothelial cells (BECs) or in isolated rat or mouse brain vessels (BV) or whole brain tissues (BT), determined by NGS. Shown are abundances (mean \pm SD) of RNA transcripts relative to $\beta$-ACTIN from at least three cell/ tissue preparations $\left({ }^{* * *} \mathrm{p}<0.001\right.$, One-Way ANOVA, Tukey's post hoc test)

end of ZIKV exposure, the i-BECs in Transwells were instantly co-stained with the vital dye CFDA and the plasma membrane dye CellMask Orange (Fig. 3b-g) confirming cell viability and intactness of cell-cell contacts.

During the exposure of $\mathrm{i}-\mathrm{BEC}$ cultured in Transwell inserts, ZIKV was able to cross the $\mathrm{BBB}$, escape at the abluminal surface of $\mathrm{i}$-BEC monolayers and infect i-NPs cultured in the companion wells below (Fig. 3h, i). Since the paracellular barrier integrity measured by sodium fluorescein permeability remained unchanged during this transmigration process, the experiment suggested that ZIKV did not cross the BBB through disrupted tight junctions. Similarly, the release of ZIKV internalized into i-BECs by cytolysis can be discounted since the viability of i-BECs was not affected following ZIKV infection (Fig. 3b, c). 

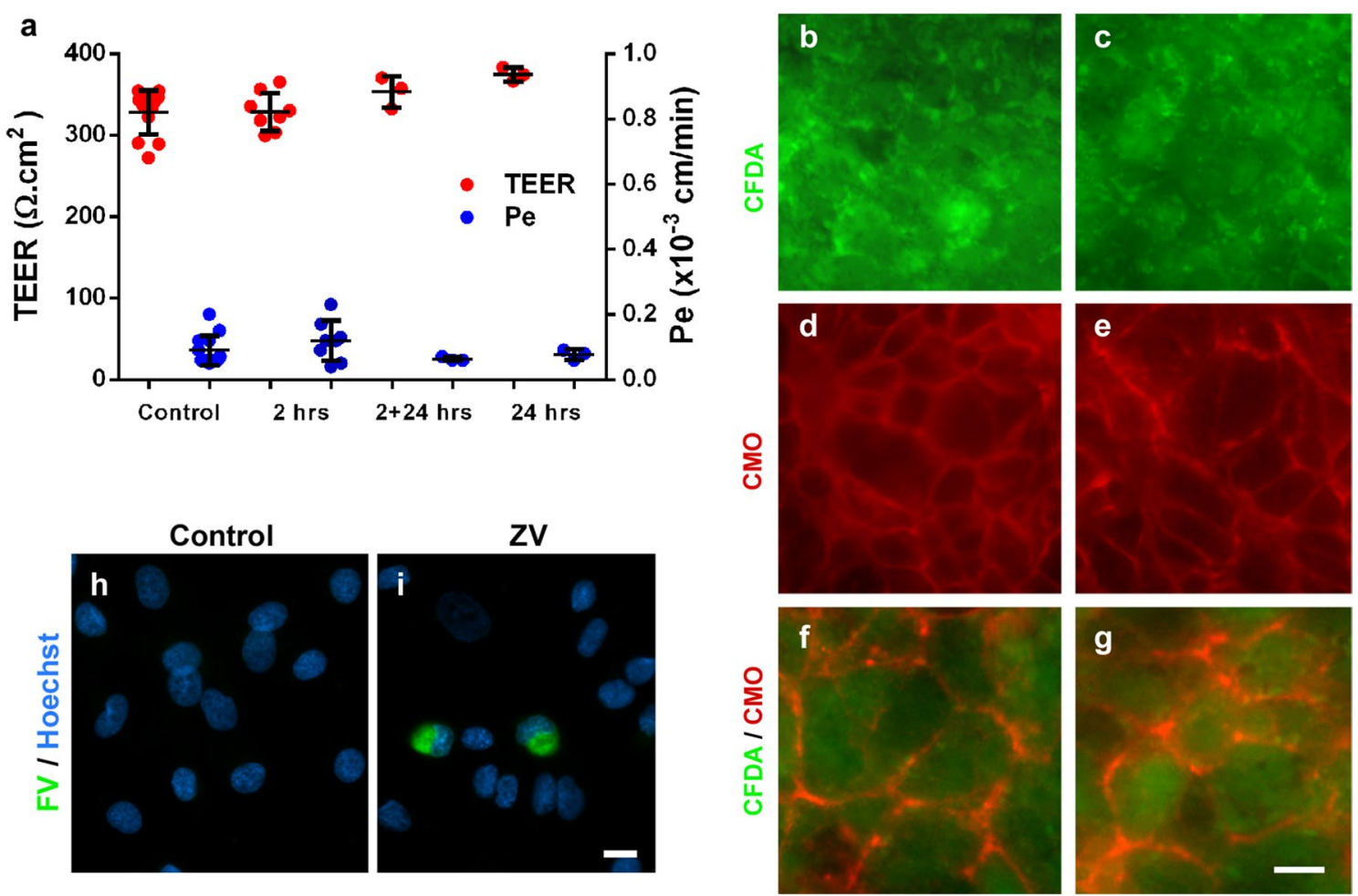

Fig. 3 ZIKV crosses the human BBB and infects i-NPs. i-BECs were cultured on Transwell inserts and the cells were mock-infected (control) or infected with ZIKV. a TEER $\left(\Omega \mathrm{cm}^{2}\right)$ was measured on each i-BEC insert, to ensure barrier integrity was intact, before infection with ZIKV. Only i-BEC inserts with TEER values $\geq 300 \Omega \mathrm{cm}^{2}$ were used in the assay. Sodium fluorescein permeability $(\mathrm{Pe} ; \mathrm{cm} / \mathrm{min}$ ) were measured in infected i-BECs at $2 \mathrm{~h}$ $(2 \mathrm{~h}), 2 \mathrm{~h}$ infection followed by $24 \mathrm{~h}$ recovery $(2+24 \mathrm{~h}$ ) or $24 \mathrm{~h}(24 \mathrm{~h})$ post ZIKV-infection with no recovery (mean \pm SD). Low sodium fluorescein impermeability (Pe) during the course of ZIKV infection, similar to mock-infected controls ( $n s, p>0.1$, One-way ANOVA), confirmed an intact barrier. To demonstrate i-BEC viability and continuity of membrane contacts, uninfected $(\mathbf{b}, \mathbf{d}, \mathbf{f})$ and ZIKV-infected $(\mathbf{c}, \mathbf{e}, \mathbf{g})$ i-BECs were co-labeled with CFDA (b, $\mathbf{c}$; green) and CellMask Orange (CMO) $(\mathbf{d}$, e; red); overlay images shown in $(\mathbf{f}-\mathbf{g})$, on the Transwell membranes at the end of ZIKV transport experiments (24 h). $\mathbf{h}, \mathbf{i}$ ZIKV crossing of the BBB and infection of underlying i-NPs (i) was confirmed through immunofluorescence staining with an anti-flavivirus antibody (FV; green) $24 \mathrm{~h}$ post-infection. Scale bar $=20 \mu \mathrm{m}$

\section{Discussion/conclusion}

This report demonstrates that ZIKV can cross the BBB formed in vitro by iPSC-derived i-BEC, i-NP and i-Ns and subsequently infect susceptible neural cells. In line with these findings, two recent studies have shown that ZIKV infects, triggers inflammatory activation and releases basolaterally from primary human brain microvascular cells (hBMECs) [26] grown in Transwell inserts, without an overt barrier disruption [27]. The drawback of these studies was low $\left(<100 \Omega \mathrm{cm}^{2}\right)$ baseline TEER of primary hBMECs, which could 'mask' subtle changes in paracellular tightness caused by harmful agents. In contrast, a 3D BBB model formed by a human BEC line grown in rotating bioreactor under flow conditions exhibited high resistance to infection by diverse RNA viruses, including ZIKV [12].

The current study confirms that ZIKV infects and crosses a Transwell human BBB model with TEER values $\geq 300 \Omega \mathrm{cm}^{2}$, without compromising its paracellular permeability for small molecule sodium fluorescein or the viability of the $\mathrm{i}-\mathrm{BEC}$ monolayer. Based on these observations, we hypothesize that the primary mechanism of ZIKV traversing the BBB is through $\mathrm{i}-\mathrm{BEC}$ infection, transcellular passage and release on the abluminal side of the monolayer. However, we cannot exclude the possibility that some viral particles could selectively modulate tight junctions and cross into the bottom chamber via paracellular diapedesis without overtly disrupting the BBB permeability; similar mechanism has been described for primed inflammatory T-cells [28]. Since ZIKV has been shown to induce inflammatory activation of brain endothelial cells [26], it might also affect other barrier properties of i-BECs, including transporter function. Several other mechanisms for neurotropic viruses to enter the CNS have been proposed [29, 30]. Notably, the ability of ZIKV to cross the placenta [31] via infected monocytes has also been demonstrated. Although transport via infected immune cells was not examined in this study, it 
remains a possible natural route of entry for ZIKV into the brain in vivo.

This study further demonstrates that the basolateral release of ZIKV from i-BEC monolayers results in the efficient infection of AXL-expressing i-NPs. Infection of $\mathrm{i}-\mathrm{Ns}$, in which AXL is down-regulated, was much lower. Susceptibility of NPs to ZIKV infection has previously been linked to AXL-mediated ZIKV entry [19, 32] leading to increased cell death, dysregulation of cell-cycle progression and attenuated growth [23]. Similar to i-NPs, ZIKV tropism for endothelial cells has also been shown to positively correlate with AXL expression [24, 25]. Since AXL ablation/inhibition in some studies did not result in reduced ZIKV infection [33, 34] other flavivirus adhesion and entry cofactors, including glycosaminoglycans, integrins and other phosphatidylserine (PS) receptors of the TIM (T cell immunoglobulin and mucin domain) and TAM (TYRO3, AXL, and MERTK) families [35], provide additional molecular context for ZIKV permissiveness of various neural cell types, and possibly BECs. In contrast to MERTK and TYRO3, AXL is highly abundant in human brain endothelial cells, including i-BECs, and is also enriched in brain microvessels compared to target brain tissue, potentially contributing to brain tropism of ZIKV. Since ZIKV infection of human BEC in this and other studies did not produce cytotoxic effects [24, 25, 27], it has been proposed that BECs could act as a viral reservoir enabling persistent ZIKV infection and replication, hindering its clearance from neuronal tissues [26].

This in vitro study demonstrates that ZIKV can cross the intact $\mathrm{BBB}$, potentially via a transcellular pathway, and infect susceptible neural cells, notably i-NPs. Understanding the mechanisms of neurotropic virus-mediated $\mathrm{BBB}$ and CNS penetration will be important in designing strategies to reduce the devastating CNS sequelae of ZIKV infection. The human iPSC-derived BBB models, in conjunction with allogeneic i-NPs and i-Ns such as used in this study, provide an important advantage for studies of bacterial [36] and viral invasion into CNS, in particular for those pathogens that have poor infectivity in rodent models and high tropism for neural cells. The model used here represents a valuable tool to aid in the development and screening of antiviral therapeutics to limit ZIKV and other viral-induced pathologies in the CNS.

\section{Methods}

\section{ZIKV strain and culturing}

Zika virus (ZIKV) was isolated in 2013 from a Canadian patient who had recently returned from Thailand [11]. The ZIKV belongs to the Asian lineage and was a gift from Dr. D. Safronetz at the National Microbiology Laboratory, Winnipeg, Manitoba. ZIKV was grown in Vero cells (ATCC CCL-81.5) with DMEM, 10\% heat inactivated fetal bovine serum (HI FBS), $100 \mathrm{U} / \mathrm{ml}$ penicillin and $100 \mu \mathrm{g} / \mathrm{ml}$ streptomycin (Pen/Strep). The cell culture supernatant was centrifuged at $1200 \times g, 10 \mathrm{~min}$, $4{ }^{\circ} \mathrm{C}$ to remove cell debris, then concentrated with a $2 \mathrm{~h}$ centrifugation at $30,000 \times g, 4{ }^{\circ} \mathrm{C}$. The pellet was re-suspended in a small volume of the supernatant plus $10 \% \mathrm{HI}$ FBS final. ZIKV was titrated on Vero E6 cells.

\section{Differentiation of iPSCs into i-BEC, i-NPs and i-Ns}

All studies with human cells and tissues have been approved by the Ottawa Hospital- and the National Research Council of Canada's Research Ethics Boards. Amniotic fluid cells (AFCs) were obtained under informed written consent at 26 weeks of gestation from a single female following routine amniocentesis. Genetic analysis revealed normal karyotypes. The AFC were cultured in DMEM supplemented with $20 \%$ FBS, as previously described [37]. Induced pluripotent stem cells (iPSCs) were generated by transfecting AFCs with oriP/EBNA1 episomal vectors EP4 E02S EN2K (Addgene-20925), pCEP4 M2L (Addgene-20926) and pEP4 E02S ET2K (Addgene-20927) using the Nucleofector I Device (Amaxa), as previously described [18]. To obtain iPSC-derived induced brain endothelial cells (i-BECs), a two-step differentiation protocol was applied, as previously described [18]. In brief, iPSCs were cultured in KnockOut DMEM/F12 medium (Life Technologies) supplemented with $20 \%$ KnockOut Serum Replacement, $1 \times$ Non-Essential Amino Acids, $1 \times$ Glutamax and $55 \mu \mathrm{M} \beta$-mercaptoethanol (all from Life Technologies) for 5-7 days after which the medium was switched to complete endothelial differentiation medium (EM) composed of human serum free endothelial medium (Life Technologies) supplemented with $1 \%$ platelet-poor plasma derived serum (PDS, Alfa-Aesar) and $20 \mathrm{ng} / \mathrm{ml}$ of bFGF (Life Technologies) for 9-10 days.

To generate iPSC derived neural progenitor cells (i-NPs), iPSCs were cultured in Matrigel coated plates in Stemdiff Neural Induction Medium (Stem Cell Technologies), according to manufacturer's instructions and maintained for an additional 3 weeks with daily changes in the Stemdiff Neural Progenitor medium (Stem Cell Technologies). Neurons (i-Ns) were differentiated from i-NP monolayers in DMEM/F12 supplemented with B27 (both from Life Technologies) with medium replenished every other day. Detailed protocols for iPSC derived i-NP and $\mathrm{i}-\mathrm{N}$ differentiation and characterization have been previously described in [18].

\section{Zika virus infection of i-BEC, i-NP, and i-Ns}

For the direct infection of i-NP and i-Ns, the virus was added to the culture medium of the cells in a 12 well plate for $24-72 \mathrm{~h}$ at an MOI=4. The mock-infected controls 
received the equivalent volume of DMEM, 10\% HI FBS and Pen/Strep without ZIKV. The infection of the i-BECs with ZIKV was conducted 5 separate times at an $\mathrm{MOI}=4$ and samples were collected for analyses at 2 or $24 \mathrm{~h}$ postinfection. The immunofluorescence staining to detect ZIKV in infected cells performed in duplicate, in at least two separate experiments. The Western blot detection of ZIKV in cell lysates was conducted in two separate experiments.

\section{Immunofluorescence staining}

i-NPs or i-Ns grown on glass coverslips were fixed for $10 \mathrm{~min}$ with $4 \%$ paraformaldehyde, washed with PBS and permeabilized for 20 min with $0.1 \%$ Triton X-100 in PBS. Cells were washed with PBS, incubated with protein block (DAKO) for $20 \mathrm{~min}$, then with primary antibodies, diluted in antibody diluent (DAKO) for $1 \mathrm{~h}$. Primary antibodies were: mouse monoclonal anti-flavivirus group antigen, (Cat \# MAB10216 Millipore), rabbit monoclonal anti-AXL (Cell Signaling Cat\#8661), mouse monoclonal anti-MAP2 (Sigma Cat \#M1406) and rabbit polyclonal anti-ZO-1 (Invitrogen Cat\#402200). Cells were washed with PBS, before adding secondary antibodies for $1 \mathrm{~h}$. Secondary antibodies were ALEXA488 conjugated goat anti-rabbit IgG (Invitrogen Cat\#A11008), ALEXA488conjugated goat anti-mouse IgG (Life Technologies A21131), Rhodamine-conjugated goat anti-rabbit IgG (Invitrogen Cat\#R6394), and Rhodamine-conjugated goat anti-mouse IgG (Invitrogen Cat\#R6393). Cells were washed with PBS and mounted with fluorescent mounting medium (DAKO) spiked with $5 \mu \mathrm{g} / \mathrm{ml}$ of Hoechst 33258. Samples were imaged on a Zeiss Axiovert fluorescence microscope with an Axiocam HRm camera using a $20 \times / 0.5$ Plan Neofluar objective and Zeiss filter set 1 (488010-0000-000), EX BP 365/12, EM LP 397 for Hoechst, EM BP 515/65 for FITC, and EX BP 546/12, EM LP 590 for TRITC. Images were analyzed with Adobe Photoshop 7.0.1. The images obtained were done in duplicate and were repeated at least once.

\section{Western blotting}

Cells were lysed in RIPA buffer (50 mM Tris, $\mathrm{pH} 7.4$, $150 \mathrm{mM} \mathrm{NaCl}, 2 \mathrm{mM}$ EDTA, 0.1\% SDS, 1\% Deoxycholate, $1 \%$ triton $\mathrm{X}-100,1 \times$ protease inhibitor (Roche) and boiled in a water bath for $5 \mathrm{~min}$ to inactivate virus. Proteins were separated by $10 \%$ SDS-PAGE and transferred to nitrocellulose. Membranes were probed with rabbit monoclonal anti-AXL (Cell Signalling), or mouse monoclonal anti-flavivirus antibodies (Millipore) and antiACTIN (BioRad) followed by HRP-conjugated secondary antibodies and were developed with Clarity ECL kit (Biorad) and imaged on a Fluorochem Q imager (Alpha Innotech). Images were analyzed in Adobe Photoshop and original scanned images are shown in Additional file 1: Figure S2.

\section{BBB model in vitro using transwell culture system}

The human i-BEC transwell BBB model was generated, as previously described [18]. Briefly, the i-BECs were dissociated with Accutase (Stem Cell Technologies) and seeded at a density of $5 \times 10^{5}$ i-BECs per $0.9 \mathrm{~cm}^{2}$ cell growth area on a Transwell insert $(1 \mu \mathrm{m}$ pore size, BDFalcon) coated with $0.5 \%$ gelatin in $1 \mathrm{ml}$ complete EM medium with $10 \mu \mathrm{M}$ Y27362 (ROCK Inhibitor, Stem Cell Technologies); $2 \mathrm{ml}$ of the same medium were added to the bottom (basal) wells of the companion plate, and incubated overnight at $37{ }^{\circ} \mathrm{C}$ in $5 \% \mathrm{CO}_{2}$. The following day, the complete EM medium, without the Rock Inhibitor, was replaced only in the apical compartment of the Transwell insert and confluent monolayer formation was assessed $48 \mathrm{~h}$ post-plating.

\section{BBB in vitro permeability assays}

Prior to being used in the BBB permeability assays, each $\mathrm{i}$-BEC insert was assessed by measuring transendothelial electrical resistance (TEER). TEER measurements were conducted on a CellZscope apparatus (Nanoanalytics) using $1 \mathrm{~cm}$ diameter electrodes and standard spectrum settings: frequency $1 \mathrm{~Hz}-100 \mathrm{kHz}$, points per decade 9 and logarithmic spacing. Final TEER values in $\Omega \mathrm{cm}^{2}$ were calculated by subtracting TEER values for the empty inserts from the TEER values for the inserts with i-BECs. The i-BEC monolayers on each insert were considered intact when the TEER values were $\geq 300 \Omega \mathrm{cm}^{2}$, as previously described [18].

To assess the ability of ZIKV to cross the BBB in vitro the virus was added to the media in the apical Transwell insert at an $\mathrm{MOI}=4$. The inoculum was left on the cells for $24 \mathrm{~h}$ except for the experiment in which the inoculum was removed after $2 \mathrm{~h}$ and replaced with complete endothelial medium for additional $24 \mathrm{~h}(2+24 \mathrm{~h})$. The inserts were placed in a 12 well companion plate containing i-NPs growing on the bottom of the well or on glass coverslips, as appropriate.

To test whether the permeability of the i-BEC monolayer was affected by ZIKV, sodium fluorescein permeability (Pe) (apical to basal) was also determined at various time points during the BBB exposure to ZIKV. The assay was conducted twice at $2 \mathrm{~h}$, once at $2+24 \mathrm{~h}$ recovery and once at $24 \mathrm{~h}$. Briefly, the i-BEC transwell inserts were washed sequentially in three consecutive wells containing $2 \mathrm{ml}$ pre-warmed Hanks Balanced Salt Solution (HBSS) to remove residual medium. The inserts were then placed into companion plates containing $2 \mathrm{ml}$ of pre-warmed transport buffer (5 mM $\mathrm{MgCl}_{2}$ and $10 \mathrm{mM}$ HEPES in HBSS, pH 7.4), equilibrated to $37{ }^{\circ} \mathrm{C}$ for $5-10 \mathrm{~min}$ and 
then $500 \mu \mathrm{l}$ of the transport buffer was carefully removed from the top (apical) chamber of each insert and replaced with $500 \mu \mathrm{l}$ of sodium fluorescein $(50 \mu \mathrm{g} / \mathrm{ml})$ in transport buffer. The plates were then incubated at $37{ }^{\circ} \mathrm{C}$ with gentle rotation $(20 \mathrm{rpm} / \mathrm{min})$, and $100 \mu \mathrm{l}$ of transport buffer was collected from the bottom (basal) companion wells at 15, 30, 45 and $60 \mathrm{~min}$ intervals for permeability analysis, as previously described [18]. Following each collection, $100 \mu \mathrm{l}$ pre-warmed transport buffer were added back to the companion wells and the plates were returned to the incubator. Inserts without cells were used for the background controls. The quantitation of sodium fluorescein in each sample was measured using a fluorescent plate reader (excitation $485 \mathrm{~nm}$ and excitation $530 \mathrm{~nm}$ ) and plotted against a standard curve $(0-50 \mathrm{ng}$ sodium fluorescein solution in transport buffer).

In addition, at the end of ZIKV exposure, i-BEC viability was assessed using $2.4 \mu \mathrm{g} / \mathrm{ml}$ CFDA staining (Invitrogen) and continuity of inter-cellular contacts was assessed using a plasma membrane stain, CellMask Orange (CMO, $5 \mu \mathrm{g} / \mathrm{ml}$ ) (Life Technologies), as previously described [18]. The cells were imaged using a $20 \times$ HMC objective on an Axiovert 200 M Microscope (Zeiss).

\section{Quantitation of AXL expression in brain endothelial cells and isolated vessels}

All studies with human cells and tissues have been approved by the National Research Council's Research Ethics Board. Three sources of primary human brain microvascular endothelial cells (hBMECs) were used: one generated in-house from normal brain tissue removed during surgery for epilepsy; Cell Systems (Cat\# ACBRI 376, mycoplasma negative); and Angio-Proteomie (Cat \#cAP-0002, mycoplasma negative). The HBMECs were pooled for further analysis. Adult rat immortalized brain endothelial cells were developed in house, as previously described [38]. The brain microvessels were isolated from rat or mouse brain tissues by sequentially passing tissue homogenates through 350, 112 and $20 \mu \mathrm{m}$ Nitex filters. The microvessels were collected from the filter-top of both 112 and $20 \mu \mathrm{m}$ filters and the flow-through was collected as the brain parenchyma fraction.

RNA was extracted from cultured HBMECs, as well as from brain microvessels and total brain tissues from three rats and mice using RNeasy Plus Mini kit (Qiagen) and NucleoSpin RNA plus kit (Macherey-Nagel GmbH \& Co. KG), respectively, according to manufacturer's instructions. Genomic DNA contamination was removed by Turbo DNA-Free Kit (Life Technologies). RNA quality was assessed using Agilent Bioanalyzer 2100. RNA-Seq Libraries were generated using the TruSeq RNA kit (Illumina), as per manufacturer's instructions. The RNA-Seq libraries were quantified by qPCR according to the Illumina Sequencing Library qPCR Quantification Guide and the quality of the libraries was evaluated on Agilent Bioanalyzer 2100 using the Agilent DNA-100 chip. The RNA-Seq library sequencing was performed using Illumina Next-Seq. RNA-Seq data was analyzed using Galaxy (useGalaxy.org).

The protein quantification was performed as follows. Membranes of cultured human brain endothelial cell line, hCMEC/D3 (obtained from Dr. Pierre-Olivier Couraud, Institute Cochin, INSERM, Paris, France), were isolated as described in [39] and protein samples prepared and analysed by nano-LC-MS/MS exactly as described in [40]. For LC-MS measurements, intensity-based absolute quantification values were calculated for AXL and $\beta$-actin, as described previously [41]. The collection of transcript and proteome data was analyzed to determine the relative abundance of AXL in specific data sets compared to the whole transcriptome or proteome.

\section{Additional file}

Additional file 1. Supplementary information.

\section{Authors' contributions}

$J A, M R-L, C S, W C$, and MB-Y designed the experiments; JA, MR-L and CS conducted the experiments; $Q \mathrm{~L}$ and $\mathrm{AH}$ contributed NGS and proteomics data; JA, MR-L, CS, AJ, DBS, WC, and MB-Y wrote the manuscript. All authors read and approved the final manuscript.

\section{Author details}

${ }^{1}$ Human Health Therapeutics Research Center, National Research Council of Canada, 100 Sussex Dr., Ottawa, ON, Canada. ${ }^{2}$ Human Health Therapeutics Research Center, National Research Council of Canada, Bldg M54-1200 Montreal Rd., Ottawa, ON K1A 0R6, Canada.

\section{Acknowledgements}

Thank you to Dr. D. Safronetz, Public Health Agency of Canada for the ZIKV isolate. We would like to thank, the late Dr. Andree Gruslin and Dr. Felipe Moretti from the Ottawa Hospital and University of Ottawa for supplying the amniotic fluid cells. Thank you to Christie Delaney and Wen Ding for proteomics analysis and to Sonia Leclerc, Dao Ly and Ewa Baumann for NGS analysis. We dedicate this paper, in memoriam, to Dr. Judie Alimonti (1960-2017) for her innovative work towards understanding ZIKV pathogenesis and to Dr. Mahmud BaniYaghoub (1964-2016) for his inspiring leadership in developing the human BBB model.

\section{Competing interests}

Her Majesty the Queen in right of Canada holds a patent on the "Human Blood-Brain Barrier Model" PCT-CA2015-051297. The authors declare that they have no competing interests.

\section{Availability of data and materials}

All relevant data and material are available from the authors.

\section{Consent for publication}

Not applicable.

\section{Ethics approval and consent to participate}

All studies with human cells and tissues have been approved by the Ottawa Hospital, and the National Research Council of Canada's Research Ethics

Boards. Amniotic fluid cells (AFCs) were obtained at 26 weeks of gestation 
from a single female from the Ottawa Hospital following routine amniocentesis. Informed written consent was obtained to use the amniotic fluid for research purposes and all the methods were carried out in accordance with the relevant guidelines and regulations as approved by the Ottawa Hospital Research Ethics Board.

\section{Funding}

This study was funded by the National Research Council of Canada.

\section{Publisher's Note}

Springer Nature remains neutral with regard to jurisdictional claims in published maps and institutional affiliations.

Received: 5 February 2018 Accepted: 30 April 2018

Published online: 15 May 2018

\section{References}

1. Lazear HM, Diamond MS. Zika virus: new clinical syndromes and its emergence in the western hemisphere. J Virol. 2016;90:4864-75. http://www. ncbi.nlm.nih.gov/pubmed/26962217. Accessed 15 Jan 2018.

2. Johansson MA, Mier-y-Teran-Romero L, Reefhuis J, Gilboa SM, Hills SL. Zika and the risk of microcephaly. N Engl J Med. 2016;375:1-4. http:// www.nejm.org/doi/10.1056/NEJMp1605367. Accessed 15 Jan 2018.

3. Tang BL. Zika virus as a causative agent for primary microencephaly: the evidence so far. Arch Microbiol; 2016. http://link.springer.com/10.1007/ s00203-016-1268-7. Accessed 15 Jan 2018.

4. Mlakar J, Korva M, Tul N, Popović M, Poljšak-Prijatelj M, Mraz J, et al. Zika virus associated with microcephaly. N Engl J Med. 2016;374:951-8. http:// www.nejm.org/doi/10.1056/NEJMoa1600651. Accessed 15 Jan 2018.

5. Dang J, Tiwari SK, Lichinchi G, Qin Y, Patil VS, Eroshkin AM, et al. Zika virus depletes neural progenitors in human cerebral organoids through activation of the innate immune receptor TLR3. Cell Stem Cell. 2016;19:258-65. https://doi.org/10.1016/j.stem.2016.04.014.

6. Li H, Saucedo-Cuevas L, Regla-Nava JA, Chai G, Sheets N, Tang W, et al. Zika virus infects neural progenitors in the adult mouse brain and alters proliferation. Cell Stem Cell. 2016;19:593-8. http://www.ncbi.nlm.nih.gov/ pubmed/27545505. Accessed 15 Jan 2018.

7. Bell TM, Field EJ, Narang HK. Zika virus infection of the central nervous system of mice. Arch Gesamte Virusforsch. 1971;35:183-93. http://link. springer.com/10.1007/BF01249709. Accessed 15 Jan 2018.

8. Shao Q, Herrlinger S, Yang SL, Lai F, Moore JM, Brindley MA, et al. Zika virus infection disrupts neurovascular development and results in postnatal microcephaly with brain damage. Development. 2016;143:4127-36. http://dev.biologists.org/lookup/doi/10.1242/dev.143768. Accessed 15 Jan 2018.

9. Da Silva IRF, Frontera JA, De Filippis AMB, Do Nascimento OJM. Neurologic complications associated with the Zika virus in Brazilian adults. JAMA Neurol. 2017;74:1190-8. http://www.ncbi.nlm.nih.gov/pubme d/28806453. Accessed 19 Jan 2018.

10. Muñoz LS, Parra B, Pardo CA. Neurological implications of Zika virus infection in adults. J Infect Dis. 2017;216:S897-905. http://fdslive.oup.com/ www.oup.com/pdf/production_in_progress.pdf. Accessed 16 Jan 2018.

11. Fonseca K, Meatherall B, Zarra D, Drebot M, MacDonald J, Pabbaraju K, et al. First case of Zika virus infection in a returning Canadian traveler. Am J Trop Med Hyg. 2014;91:1035-8. http://www.ncbi.nlm.nih.gov/pubme d/25294619. Accessed 16 Jan 2018.

12. Bramley JC, Drummond CG, Lennemann NJ, Good CA, Kim KS, Coyne CB. A three-dimensional cell culture system to model RNA virus infections at the blood-brain barrier. mSphere. 2017;2:e00206-17. http://www.ncbi. nlm.nih.gov/pubmed/28656176. Accessed 17 Jan 2018.

13. Dahm T, Rudolph H, Schwerk C, Schroten H, Tenenbaum T. Neuroinvasion and inflammation in viral central nervous system infections. Mediat Inflamm. 2016;2016:8562805. http://www.ncbi.nIm.nih.gov/pubme d/27313404. Accessed 18 Jan 2018.

14. Rendelman B. In vitro and in vivo blood-brain barrier models to study west Nile virus pathogenesis. Methods Mol Biol. 2016;1435:61-9. http:// www.ncbi.nlm.nih.gov/pubmed/27188553. Accessed 18 Jan 2018.
15. Stanimirovic DB, Bani-Yaghoub M, Perkins M, Haqqani AS. Blood-brain barrier models: in vitro to in vivo translation in preclinical development of CNS-targeting biotherapeutics. Expert Opin Drug Discov. 2015;10:141-55. http://www.tandfonline.com/doi/full/10.1517/17460441.2015.974545. Accessed 15 Jan 2018.

16. Helms HC, Abbott NJ, Burek M, Cecchelli R, Couraud PO, Deli MA, et al. In vitro models of the blood-brain barrier: an overview of commonly used brain endothelial cell culture models and guidelines for their use. J Cereb Blood Flow Metab. 2016;36:862-90. http://www.ncbi.n/m.nih.gov/ pubmed/26868179. Accessed 23 Jan 2018.

17. Lippmann ES, Azarin SM, Kay JE, Nessler RA, Wilson HK, Al-Ahmad A, et al. Human blood-brain barrier endothelial cells derived from pluripotent stem cells. Nat Biotechnol. 2012;30:2014. http://www.ncbi.nlm.nih.gov/ pubmed/22729031. Accessed 15 Jan 2018.

18. Ribecco-Lutkiewicz M, Sodja C, Haukenfrers J, Haqqani AS, Ly D, Zachar P, Baumann E, Ball M, Huang J, Rukhlova M, Martina M, Liu Q, Stanimirovic D, Jezierski A, Bani-Yaghoub M. A novel human induced pluripotent stem cell blood-brain barrier model: applicability to study antibody-triggered receptor-mediated transcytosis. Sci Rep. 2018;In press:1873. http://www. nature.com/articles/s41598-018-19522-8. Accessed 31 Jan 2018.

19. Nowakowski TJ, Pollen AA, Di Lullo E, Kriegstein AR, Francisco S, Francisco $\mathrm{S}$, et al. Expression analysis highlights $\mathrm{AXL}$ as a candidate Zika virus entry receptor in human stem cells. Cell Stem Cell. 2017;18:591-6.

20. Perera-Lecoin $M$, Meertens $L$, Carnec $X$, Amara A. Flavivirus entry receptors: an update. Viruses. 2013;6:69-88. http://www.mdpi.com/19994915/6/1/69/. Accessed 15 Jan 2018.

21. Meertens L, Labeau A, Dejarnac O, Cipriani S, Sinigaglia L, Bonnet-Madin $L$, et al. AxI mediates ZIKA virus entry in human glial cells and modulates innate immune responses. Cell Rep. 2017;18:324-33. https://www.cell. com/cell-reports/fulltext/S2211-1247(16)31752-1?_returnURL=https \%3A\%2F\%2Flinkinghub.elsevier.com\%2Fretrieve\%2Fpii\%2FS2211124 716317521\%3Fshowall\%3Dtrue. Accessed 15 Jan 2018.

22. Nowakowski TJ, Pollen AA, Di Lullo E, Sandoval-Espinosa C, Bershteyn M, Kriegstein AR. Expression analysis highlights AXL as a candidate Zika virus entry receptor in neural stem cells. Cell Stem Cell. 2016;18:591-6. https:// doi.org/10.1016/j.stem.2016.03.012.

23. Tang H, Hammack C, Ogden SC, Wen Z, Qian X, LiY, et al. Zika virus infects human cortical neural progenitors and attenuates their growth. Cell Stem Cell. 2016;18:587-90. http://www.sciencedirect.com/science/article/pii/ S1934590916001065?via\%3Dihub. Accessed 19 Jan 2018.

24. Liu S, DeLalio LJ, Isakson BE, Wang TT. AXL-mediated productive infection of human endothelial cells by Zika virus novelty and significance. Circ Res. 2016;119:1183-9. http://www.ncbi.nlm.nih.gov/pubmed/27650556. Accessed 16 Jan 2018.

25. Richard AS, Shim BS, Kwon YC, Zhang R, Otsuka Y, Schmitt K, et al. AXL-dependent infection of human fetal endothelial cells distinguishes Zika virus from other pathogenic flaviviruses. Proc Natl Acad Sci. 2017;114:2024-9. http://www.pnas.org/lookup/doi/10.1073/pnas.16205 58114. Accessed 15 Jan 2018.

26. Mladinich MC, Schwedes J, Mackow ER. Zika virus persistently infects and is basolaterally released from primary human brain microvascular endothelial cells. MBio. 2017;8:11. http://mbio.asm.org/content/8/4/ e00952-17 abstract Accessed 15 Jan 2018

27. Papa MP, Meuren LM, Coelho SVA, de Oliveira Lucas CG, Mustafá YM, Lemos Matassoli F, et al. Zika virus infects, activates, and crosses brain microvascular endothelial cells, without barrier disruption. Front Microbiol. 2017;8:2557. https://www.ncbi.nlm.nih.gov/pmc/articles/PMC57 43735/. Accessed 15 Jan 2018.

28. Coisne C, Lyck R, Engelhardt B. Live cell imaging techniques to study $T$ cell trafficking across the blood-brain barrier in vitro and in vivo. Fluids Barriers CNS. 2013;10:7. http://www.ncbi.nlm.nih.gov/pubmed/23336847. Accessed 5 Apr 2018.

29. Kim K, Shresta S. Neuroteratogenic viruses and lessons for Zika virus models. Trends Microbiol. 2016:622-36. http://www.sciencedirect.com/scien ce/article/pii/S0966842X16300658?via\%3Dihub. Accessed 15 Jan 2018.

30. Doran KS, Banerjee A, Disson O, Lecuit M. Concepts and mechanisms: crossing host barriers. Cold Spring Harb Perspect Med. 2013;3. http:// www.ncbi.nlm.nih.gov/pubmed/23818514. Accessed 15 Jan 2018.

31. Quicke KM, Bowen JR, Johnson EL, McDonald CE, Ma H, O'Neal JT, et al. Zika virus infects human placental macrophages. Cell Host Microbe. 
2016;20:83-90. http://www.ncbi.nlm.nih.gov/pubmed/27247001. Accessed 15 Jan 2018

32. Lin MY, Wang YL, Wu WL, Wolseley V, Tsai MT, Radic V, et al. Zika virus infects intermediate progenitor cells and post-mitotic committed neurons in human fetal brain tissues. Sci Rep. 2017;7:14883. http://www.natur e.com/articles/s41598-017-13980-2. Accessed 16 Jan 2018.

33. Li F, Wang PR, Qu LB, Yi CH, Zhang FC, Tang XP, et al. AXL is not essential for Zika virus infection in the mouse brain. Emerg Microbes Infect. 2017;6:e16. http://www.nature.com/articles/emi201710. Accessed 19 Jan 2018

34. Wells MF, Salick MR, Wiskow O, Ho DJ, Worringer KA, Ihry RJ, et al. Genetic ablation of AXL does not protect human neural progenitor cells and cerebral organoids from Zika virus infection. Cell Stem Cell. 2016;19:703-8. http://www.sciencedirect.com/science/article/pii/S19345909163040 76?via\%3Dihub. Accessed 19 Jan 2018.

35. Sirohi D, Kuhn RJ. Zika virus structure, maturation, and receptors. J Infect Dis. 2017:S935-44. https://academic.oup.com/jid/article/216/suppl_10/ 5935/4753686. Accessed 5 Apr 2018.

36. Kim BJ, Bee OB, McDonagh MA, Stebbins MJ, Palecek SP, Doran KS, et al. Modeling group B Streptococcus and blood-brain barrier interaction by using induced pluripotent stem cell-derived brain endothelial cells. mSphere. 2017;2. http://www.ncbi.nlm.nih.gov/pubmed/29104935. Accessed 5 Apr 2018.
37. Jezierski A, Gruslin A, Tremblay R, Ly D, Smith C, Turksen K, et al. Probing stemness and neural commitment in human amniotic fluid cells. Stem Cell Rev Rep. 2010;6:199-214. http://www.ncbi.nlm.nih.gov/pubme d/20221716. Accessed 22 Jan 2018.

38. Muruganandam A, Herx LM, Monette R, Durkin JP, Stanimirovic DB. Development of immortalized human cerebromicrovascular endothelial cell line as an in vitro model of the human blood-brain barrier. FASEB J. 1997;11:1187-97. http://www.ncbi.nlm.nih.gov/pubmed/9367354. Accessed 12 Apr 2018.

39. Haqqani AS, Hill JJ, Mullen J, Stanimirovic DB. Methods to study glycoproteins at the blood-brain barrier using mass spectrometry. Methods Mol Biol. 2011;686:337-53. http://link.springer.com/10.1007/978-1-60761 -938-3_16. Accessed 15 Jan 2018.

40. Haqqani AS, Kelly JF, Stanimirovic DB. Quantitative protein profiling by mass spectrometry using label-free proteomics. Methods Mol Biol. 2008:439:241-56. http://link.springer.com/10.1007/978-1-59745-1888_17. Accessed 15 Jan 2018

41. Ahrné E, Molzahn L, Glatter T, Schmidt A. Critical assessment of proteome-wide label-free absolute abundance estimation strategies. Proteomics. 2013:13:2567-78. http://www.ncbi.nlm.nih.gov/pubmed/23794 183. Accessed 5 Apr 2018
Ready to submit your research? Choose BMC and benefit from:

- fast, convenient online submission

- thorough peer review by experienced researchers in your field

- rapid publication on acceptance

- support for research data, including large and complex data types

- gold Open Access which fosters wider collaboration and increased citations

- maximum visibility for your research: over 100M website views per year

At BMC, research is always in progress.

Learn more biomedcentral.com/submissions 